\title{
Prevalencia de accidentes de tránsito no fatales en México: resultados de la ENSANUT 2006
}

\author{
Leticia Ávila-Burgos, DSc, ${ }^{(1)}$ Carlo E Medina-Solís, MSc, ${ }^{(1,2)}$ Ricardo Pérez-Núñez, MSc, ${ }^{(1)}$ Martha Hijar-Medina, DSc, ${ }^{(3)}$ Belkis \\ Aracena-Genao, MSc, ${ }^{(1)}$ Elisa Hidalgo-Solórzano, MSc, (1) Oswaldo Palma-Coca,Act. ${ }^{(4)}$
}

\author{
Ávila-Burgos L, Medina-Solís CE, Pérez-Núñez R, \\ Hijar-Medina M,Aracena-Genao B, \\ Hidalgo-Solórzano E, Palma-Coca O. \\ Prevalencia de accidentes de tránsito no fatales \\ en México: resultados de la ENSANUT 2006. \\ Salud Publica Mex 2008;50 supl I:S38-S47.
}

\section{Resumen}

Objetivo. Determinar la prevalencia y distribución de los accidentes de tránsito no fatales (ATNF) en México. Material y métodos. Se utilizaron datos de la ENSANUT 2006. En los hogares visitados se seleccionó a un adulto, un adolescente y un niño, hasta conformar una muestra de 94197 sujetos que representa a la N de 102886482 individuos. La variable dependiente fue la prevalencia de ATNF en los 12 meses anteriores a la encuesta. Resultados. La prevalencia de accidentes (de tránsito o no) fue de $6.0 \%$ y de esa cifra $16.7 \%$ correspondió a ATNF. Los hombres del grupo de 20 a 44 años, los habitantes de áreas urbanas y los de mejor nivel socioeconómico (NSE) presentaron mayor prevalencia $(p<0.05)$. Jalisco,Aguascalientes y Sonora registraron la mayor prevalencia y Guerrero, Michoacán y Oaxaca la menor. Conclusiones. Los ATNF en México se concentran en hombres de edad productiva en zonas urbanas y se relacionan con el NSE individual y el desarrollo económico de cada estado.

Palabras clave: accidente de tránsito; accidentes no fatales; prevalencia; morbilidad; mortalidad; México

\author{
Ávila-Burgos L, Medina-Solís CE, Pérez-Núñez R, \\ Hijar-Medina M,Aracena-Genao B, \\ Hidalgo-Solórzano E, Palma-Coca O. \\ Prevalence of non-fatal road traffic injuries \\ in Mexico: results from ENSANUT 2006. \\ Salud Publica Mex 2008;50 suppl I:S38-S47.
}

\begin{abstract}
Objective. To determine non-fatal road traffic injuries (NFRTI) prevalence and its distribution in Mexico. Material and Methods. Data from ENSANUT Survey 2006 were used. Using simple random sampling, one adult, one adolescent and one child were selected in each household, constituting a final sample of 94197 representing an N of 102886482 people. The dependent variable was the prevalence of road traffic injuries (RTI) during the 12 months prior to the survey. Results. The general accident prevalence was $6.0 \% ; 16.7 \%$ corresponded to NFRTI. Men in the 20 to 44 age group living in urban areas and with high socioeconomic status had a higher RTI prevalence $(p<0.05)$. Jalisco, Aguascalientes and Sonora were states with the highest prevalence of RTI, while Guerrero, Michoacan and Oaxaca were those with the lowest. Conclusions. NFRTI are frequent in Mexico and they are concentrated among men in productive ages in urban areas; they are associated with socioeconomic status at the individual level and with the state's development at the population level.
\end{abstract}

Key words: road traffic injuries; non-fatal road traffic injuries; prevalence; morbidity; mortality; Mexico

(I) Centro de Investigación en Sistemas de Salud del Instituto Nacional de Salud Pública. Cuernavaca, Morelos, México.

(2) Instituto de Ciencias de la Salud de la Universidad Autónoma del Estado de Hidalgo. Pachuca, Hidalgo, México.

(3) Centro de Investigación en Salud Poblacional del Instituto Nacional de Salud Pública. Cuernavaca, Morelos, México.

(4) Centro de Evaluación y Encuestas del Instituto Nacional de Salud Pública. Cuernavaca, Morelos, México.

Fecha de recibido: 26 de abril de 2007 - Fecha de aceptado: 4 de diciembre de 2007 Solicitud de sobretiros: MSc. Carlo E. Medina-Solis. Privada de Altillo s/n entre Av. Central y Pedro Moreno. Col. San José. 24040 Campeche, Campeche, México. Correo electrónico: cemedinas@yahoo.com 
L os accidentes de tránsito ${ }^{1}$ constituyen un importante problema de salud pública. Sus consecuencias representan una pesada carga en términos de morbimortalidad. La Organización Mundial de la Salud (OMS) calculó que en 2000 más de $2.2 \%$ de la mortalidad mundial se relacionó con los accidentes de tránsito (AT). Esto equivale a 1.2 millones de personas y se prevé que cada año alrededor de 50 millones de individuos resultarán lesionados por este tipo de suceso. Se calcula que en el mundo el promedio diario de defunciones por esta causa es de $3000 .^{2-4}$

Los accidentes constituyen una de las principales causas de muerte en México y de éstas casi $40 \%$ es atribuible a AT. Estas cifras convierten a las lesiones secundarias a AT en el tercer lugar dentro de las principales causas de muerte en la última década y representan la primera causa de muerte en la población en edad productiva $^{4-6}$ y la segunda de orfandad. ${ }^{7}$

Una característica notable de los AT es su localización: 77\% tiene lugar en zonas urbanas y los más afectados son los hombres de 15 a 29 años de edad. ${ }^{7}$ En términos de gravedad, los afectados de modo más grave son los peatones y los motociclistas, quienes además de sufrir una mayor estancia hospitalaria (6.1 y 4.9 días de estancia hospitalaria en promedio en comparación con 1.5 días en ciclistas o 2.4 días en ocupantes de vehículo de motor) presentan mayor letalidad con cifras que superan $4 \%$ del total de egresos hospitalarios por dichas causas. ${ }^{8}$ Además, cada año se producen más de 18000 incapacidades permanentes. ${ }^{9}$

No obstante, las pérdidas sociales no sólo se limitan al número de vidas perdidas o la presencia de la discapacidad temporal o permanente, sino que imponen también una gran carga para el sistema de salud, ya que es necesario consumir onerosos recursos para atender a los lesionados. En el informe mundial sobre prevención de los traumatismos por el tránsito, que publicó la OMS en 2004, se señala que por cada persona que muere a causa de lesiones se hospitaliza en promedio a 30 personas y 300 reciben atención en los servicios de urgencias, ${ }^{4,7,8}$ Se calcula que en los países de ingresos bajos y medios, como México, las lesiones por el tránsito son el origen de 30 a $86 \%$ del total de admisiones por traumatismo en los establecimientos de salud. Si a esto se suma que $43 \%$ de los individuos que sufren lesiones en accidentes de tránsito tarda en promedio un año en reincorporarse a la actividad laboral, sus consecuencias sobre la economía de los países son catastróficas. ${ }^{10}$

Una serie de elementos impide visualizar la verdadera magnitud de esta problemática. Hay que mencionar la multiplicidad de sectores participantes, ${ }^{7}$ en los cuales convergen las autoridades policiales y de tránsito y el sistema de salud, entre otros. Otro elemento importante es la complejidad de los factores que originan los AT (factores de riesgo individual, ambiente físico, características del vehículo, etc.); y, por último, el desconocimiento de la cantidad real del número de lesionados por AT. Hasta ahora, el cálculo de las tasas de AT se basa en el análisis de los registros de mortalidad, lo que contribuye a subestimar en grado considerable el problema, dado que estos cálculos no consideran a los individuos que demandan atención como efecto de los accidentes de tránsito no fatales. Además, aunque los registros hospitalarios también contribuyen a la generación de información sobre $\mathrm{AT}$, el registro de la causa externa que origina las lesiones es un hecho reciente $y$ hasta el momento es una fuente poco confiable de información. Por último, se desconoce también el porcentaje de población que sufre un AT y que no acude a buscar atención en los servicios de salud.

Ante la gravedad de este problema, es imprescindible contar con información que muestre en su justa dimensión el efecto de los AT en la sociedad mexicana. El objetivo de este trabajo fue determinar la prevalencia, describir la distribución por sexo, grupo de edad y entidad federativa, así como identificar algunos factores de riesgo vinculados con los accidentes de tránsito no fatales (ATNF) en México.

\section{Material y métodos}

\section{Diseño general y selección de la muestra}

Se analiza el componente de accidentes incluido en la Encuesta Nacional de Salud y Nutrición (ENSANUT 2006) que corresponde a un diseño transversal y recoge información de ese suceso ocurrido 12 meses antes de la aplicación de la encuesta. El diseño de ésta es probabilístico, polietápico, estratificado y por conglomerados. En la encuesta se recabó información sobre el estado de salud y nutrición de la población mexicana, la calidad y respuesta de los servicios de salud, las políticas y programas que inciden en la salud poblacional y el gasto en salud de los hogares. Su estructura permite el cálculo de indicadores con representatividad nacional, estatal y para las áreas urbanas y rurales y hace posible además diferenciar por estrato socioeconómico y principales grupos poblacionales. ${ }^{11}$

Para cumplir con sus objetivos, el muestreo de la ENSANUT 2006 se diseñó para permitir la cuantificación de prevalencias de $8.1 \%$, en el caso de las entidades federativas, y de $0.4 \%$ para el plano nacional, con la misma precisión y confianza. Además, dado que la encuesta debería posibilitar cálculos estatales con un error relativo máximo de $25 \%$, una confianza de $95 \%$, una tasa de no respuesta de $20 \%$ y un efecto de diseño 
de 1.7, se determinó un tamaño de muestra de unos 1476 hogares por estado. Se visitó a un total de 48600 viviendas y en cada una se aplicó la encuesta a todos los hogares identificados. * Siempre que la composición del hogar lo permitió mediante muestreo aleatorio simple se seleccionó a un adulto, un adolescente, un niño y un utilizador de servicios de salud (que pudo ser o no alguno de los anteriores). En el caso de la información sobre los menores de edad, el informante fue el cuidador/responsable de dicho sujeto. Para los fines del presente análisis se consideraron los cuestionarios de niños, adolescentes y adultos, lo cual conformó una muestra total de 94302 individuos. La descripción detallada de la metodología empleada puede consultarse en el informe de la encuesta. ${ }^{11}$

\section{Variables incluidas en el estudio}

En este estudio se alude al autorreporte de accidentes de tránsito no fatales, esto es, los que no causaron la muerte. La prevalencia de accidentes en los 12 meses anteriores al estudio se calculó con base en dos preguntas de la encuesta: ¿sufriste algún daño a tu salud a causa de un accidente en los últimos 12 meses?; si la persona respondía de manera afirmativa, se le preguntaba: ¿cómo te accidentaste? De las 16 opciones, tres se relacionaban con accidentes de tránsito: a) choque de o entre vehículos de transporte, b) atropellamiento y c) otros accidentes de transporte.

Las variables sociodemográficas a través de las cuales se realizó el análisis de distribución fueron: edad (dividida en menores de 10 años, 10 a 19 años, 20 a 44 años, 45 a 64 años y 65 y más años); sexo, nivel socioeconómico (NSE), entidad federativa, región geográfica (el país se dividió de manera arbitraria en cuatro regiones geográficas ${ }^{12}$ ) y como estrato rural (menor de 2500 habitantes) y urbano (2 500 y más habitantes). La metodología para la construcción del NSE se basó en un trabajo previo realizado para la Comisión de Protección Social en Salud con el fin de clasificar por deciles de ingreso a las familias que solicitan su incorporación al Seguro Popular, la cual consideró la pobreza alimentaria y patrimonial. Esta metodología se modificó tanto en función de la información disponible como de las

\footnotetext{
* Hogar: conjunto de personas relacionadas por algún parentesco o no que habitualmente duermen en una vivienda bajo el mismo techo beneficiándose de un ingreso común aportado por uno o más de los miembros del hogar. Por tanto puede haber más de un hogar por vivienda.
}

necesidades específicas del análisis de la ENSANut. La clasificación se realizó a partir de una cuantificación por fases mediante modelos tipo logit y logit ordenado. Por último, el NSE se dividió en cuatro categorías (el nivel I fue el más pobre).

\section{Análisis estadístico}

Para la realización del análisis estadístico se utilizó el modulo "svy" para muestras complejas del paquete estadístico STATA 9.2. En primer lugar se calcularon medidas de tendencia central y de dispersión, para las variables continuas, y de frecuencias y porcentajes, para las variables categóricas. En el análisis se realizaron varias comparaciones de la variable dependiente a partir de dos categorizaciones; la primera tuvo tres divisiones: a) si el sujeto refirió no haber tenido algún accidente; b) si registró algún accidente que no fuera de tránsito (NAT); y c) si notificó algún AT. Por su parte, la segunda tuvo cinco: a) ningún accidente; b) accidente que no fue de tránsito (NAT); c) choque entre vehículos de motor (AT tipo 1); d) atropellamientos (AT tipo 2); y e) otros AT (AT tipo3). Se emplearon estas categorizaciones para explorar las relaciones en conjunto y por separado de los AT.

Se usaron pruebas de la ji cuadrada para comparar las proporciones de accidentados por las diversas categorías de las variables independientes. Para adecuar la prueba al diseño de la encuesta se convirtió el estadístico Pearson chi-squared a un estadístico F, para lo cual se utilizó una corrección de segundo orden de Rao y Scott. ${ }^{13} \mathrm{El}$ análisis también incluyó la realización de un mapa en el que se muestra la distribución de los AT por estados en México. Además, se llevó a cabo un análisis de tendencia no paramétrica para cada categoría de accidente (en comparación con los sujetos sin accidentes) a través del NSE.

La fuerza de la relación entre las variables analizadas se calculó mediante razones de momios crudas y ajustadas con intervalos de confianza al 95 por ciento. La variable dependiente en ese análisis se categorizó como 0 si el sujeto señaló no haber tenido algún accidente y 1 si la persona sufrió algún AT. Se empleó el modelo de regresión logística binaria. Dado que las variables planteadas de manera inicial resultaron significativas con un valor de $p<0.20$ en el análisis bivariado, ${ }^{14}$ todas se incluyeron en el modelo multivariado.

\section{Resultados}

Se contó con información completa para 94197 individuos (99.95\%), que representan el total de la población ( $N=102886$ 482). Las características de los sujetos incluidos en el estudio se muestran en el cuadro I; 
todos los resultados registrados son ponderados. ${ }^{*} \mathrm{El}$ promedio de edad del total de entrevistados fue de $28.72 \pm 20.60$ años, $52.5 \%$ corresponde a mujeres y $76 \%$ se ubica en el área urbana. Los resultados arrojan una prevalencia de accidentes en general (de tránsito o no) de 6\% (IC95\%=5.6-6.3; N=6 130 875); de éstos, los AT representaron $16.7 \%(I C 95 \%=14.9-18.6 ; N=1026892)$ (figura 1). De los que señalaron haber sufrido algún tipo de AT, $19.3 \%$ no recibió atención.

La figura 2 presenta la distribución geográfica de los AT en el ámbito nacional; la prevalencia fue de $10 \mathrm{x}$ 1 000. Los estados con mayor prevalencia fueron Jalisco (15.1 x 1000$)$, Aguascalientes (14.7 x 1000$)$ y Sonora (14.4 x 1 000), mientras que Guerrero, Michoacán y Oaxaca tuvieron la menor $(4.1,6.6$ y 6.9 x 1000 , respectivamente).

En el análisis estadístico se usaron como categorías de la variable dependiente: a) si el sujeto notificó la ausencia de algún AT; b) si comunicó algún accidente que no fuera de tránsito (NAT); y c) si refirió algún AT. Se observaron diferencias significativas $(p<0.01)$ en cuanto a edad, sexo, zona y nivel socioeconómico. El grupo de 20 a 44 años presentó la más alta prevalencia $(1.3 \%)$ y al mismo tiempo el porcentaje más bajo de NAT $(3.7 \%)(p<0.0001)$. Los hombres mostraron mayor afectación en ambos tipos de accidentes que las mujeres (1.3 contra $0.8 \%$ para los AT y 6.2 contra 3.9 para los NAT) $(p<0.0001)$, mientras que los individuos que viven en zonas urbanas se afectaron más por los AT respecto de quienes residen en zonas rurales $(1.1 \%$ contra $0.6 \%$; $p<0.01)$. La distribución de los accidentes no atribuibles al tránsito no mostró diferencias relacionadas con el nivel socioeconómico.

Asimismo, se observó una mayor frecuencia de AT en el nivel socioeconómico más alto y se reconoció además una tendencia positiva (NP Trend $=p<0.001$ ) en la que a medida que el nivel socioeconómico aumenta lo hace también la frecuencia de AT. Otro factor utilizado para explicar la prevalencia de AT fue el índice de marginación. Se encontró una relación negativa: a menor marginación de los estados, es decir, mayor desarrollo, la prevalencia de $\mathrm{AT}$ aumentó ( $p<0.01$; figura 2 ). La zona sur del país presentó el menor porcentaje de AT $(0.85 \%)$ y el mayor porcentaje de NAT (5.12\%); no obstante, estas diferencias no fueron estadísticamente significativas $(p>0.05)$.

\footnotetext{
* El ponderador se calculó a partir de los mecanismos del muestreo complejo utilizados para obtener las cuantificaciones correspondientes en los planos nacional y estatal.
}

Cuadro I

\section{Características generales de la población INCLUIDA EN LA ENSANUT 2006, MÉxico (RESULTADOS PONDERADOS)}

\begin{tabular}{lccc}
$\begin{array}{l}\text { Variable } \\
\text { Edad }\end{array}$ & $n$ & $N$ & Media $\pm D E$ \\
& 94197 & 102886482 & $28.72 \pm 20.60$ \\
$\begin{array}{l}\text { Sexo } \\
\text { Mujeres }\end{array}$ & $n$ & $N$ & Porcentaje \\
\hline Hombres & 49795 & 54030770 & 52.5 \\
$\begin{array}{l}\text { Estrato } \\
\quad \text { Rural }\end{array}$ & 44402 & 48855712 & 47.5 \\
\hline Urbano & & & \\
& 25636 & 24186555 & 23.5 \\
\hline 68561 & 78699927 & 76.5
\end{tabular}

Nivel socioeconómico

\begin{tabular}{lrrr} 
I más bajo & $4 \mid 749$ & 42689412 & $4 \mid .5$ \\
\hline 2 & $2027 \mid$ & $255 \mid$ I 024 & 24.8 \\
\hline 3 & 23788 & 25037776 & 24.3 \\
\hline 4 más alto & 8387 & 9646779 & 9.4
\end{tabular}

Estado

\begin{tabular}{|c|c|c|c|}
\hline Aguascalientes & 3497 & $105 \mid 550$ & 1 \\
\hline Baja California & 2431 & 2835250 & 2.8 \\
\hline Baja California Sur & 2829 & 440315 & 0.4 \\
\hline Campeche & 2978 & 750926 & 0.7 \\
\hline Coahuila & 2835 & 2473485 & 2.4 \\
\hline Colima & 2901 & 562353 & 0.5 \\
\hline Chiapas & 2982 & 4251079 & 4.1 \\
\hline Chihuahua & 2833 & 3233813 & 3.1 \\
\hline Distrito Federal & 2358 & 8661282 & 8.4 \\
\hline Durango & 3050 & 485925 & 1.4 \\
\hline Guanajuato & 3076 & 4885700 & 4.8 \\
\hline Guerrero & 3048 & 3113958 & 3 \\
\hline Hidalgo & 2897 & 2331190 & 2.3 \\
\hline Jalisco & 3117 & 6649718 & 6.5 \\
\hline México Estado de & 3014 & 14151235 & 13.8 \\
\hline Michoacán & 2955 & 3978160 & 3.9 \\
\hline Morelos & 3149 & 1600367 & 1.6 \\
\hline Nayarit & 3108 & 941701 & 0.9 \\
\hline Nuevo León & 2910 & 4159335 & 4 \\
\hline Oaxaca & 2894 & 3517742 & 3.4 \\
\hline Puebla & 2773 & 5375314 & 5.2 \\
\hline Querétaro & 2967 & 1590064 & 1.6 \\
\hline Quintana Roo & 3151 & $1|33| 55$ & I.I \\
\hline San Luis Potosí & 3034 & 2408278 & 2.3 \\
\hline Sinaloa & 3078 & 2608497 & 2.5 \\
\hline Sonora & 2768 & 2383692 & 2.3 \\
\hline Tabasco & 3129 & 2012251 & 2 \\
\hline Tamaulipas & 2552 & 3008843 & 2.9 \\
\hline Tlaxcala & 3152 & 1057382 & I \\
\hline Veracruz & 2772 & 7073542 & 6.9 \\
\hline Yucatán & 3135 & 1803263 & 1.8 \\
\hline Zacatecas & 2824 & | 357 || & 1.3 \\
\hline \multicolumn{4}{|l|}{ Región } \\
\hline Norte & 22208 & 20020658 & 19.5 \\
\hline Centro & 36720 & 41784740 & 40.6 \\
\hline Distrito Federal & 2358 & 8661282 & 8.4 \\
\hline Sur & 32911 & 32419802 & 31.5 \\
\hline
\end{tabular}





Fuente: ENSANUT 2006

Figura I. Gráfica QUe ilustra la prevalencia de acciDENTES Y de ACCIDENTES de tRÁNSito en MéXICO EN EL AÑo 2006

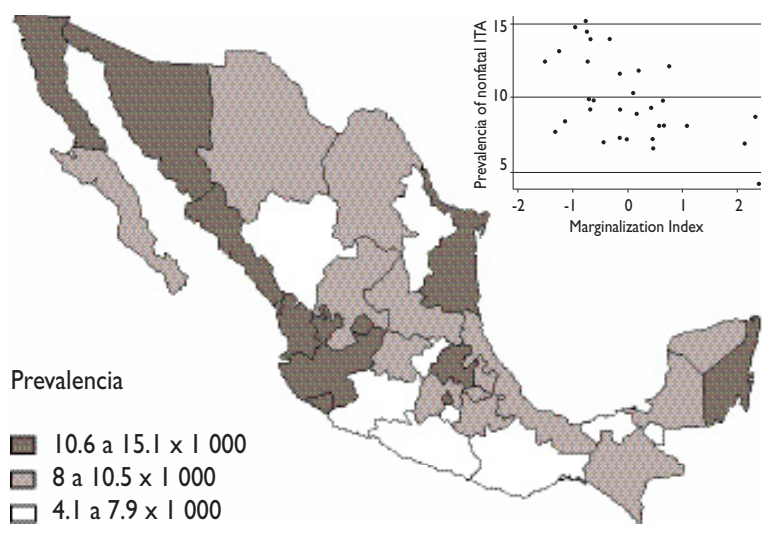

Prevalencia nacional: $10 \times 1000$

Fuente: ENSANUT 2006

Figura 2. Distribución geográfica de los accidentes de tránsito en México en 2006

En el cuadro II se presenta el análisis de diferencia de proporciones de accidentes a través de las diferentes variables de interés y categoriza los accidentes de la siguiente manera: $a)$ ningún accidente, $b$ ) accidente que no fue de tránsito (NAT), c) choque entre vehículos de motor (AT tipo 1), d) atropellamientos (AT tipo 2) y e) otros AT (AT tipo 3). Los AT más frecuentes fueron los de tipo $1(0.59 \%$; $N=603541)$, seguidos por los de tipo 2 $(0.23 \% ; N=233454)$ y los de tipo $3(0.18 \%$; $N=189897)$. El análisis por grupo de edad mostró que los de 20 a 44 años presentan el mayor porcentaje $(0.92 \%)$ de AT tipo 1 . Por su parte, los "atropellados" y los que informaron "otro accidente de tránsito" pertenecen en especial al grupo de 65 años y más $(p<0.0001)$. Se encontró además que los hombres son más propensos a presentar AT en cualquiera de sus modalidades (tipos 1,2 o 3$)(p<0.0001)$. Al considerar el lugar de ocurrencia para todos los tipo de AT, se observó que la mayor parte se presenta en la zona urbana $(p<0.05)$. En relación con la región, no se reconocieron diferencias significativas cuando se comparó por tipo de AT. Se advirtió que la prevalencia de "choque entre vehículos de motor" tiende a incrementarse conforme aumenta el nivel socioeconómico y oscila entre 0.38 en el más bajo y $1.40 \%$ en el más alto $(p<0.001)$. Por el contrario, cuanto más bajo sea el nivel socioeconómico más alto es el porcentaje de atropellados, si bien esta tendencia fue marginalmente significativa $(p=0.079)$.

En el cuadro III se muestran los resultados del análisis bivariado de regresión logística binaria y en él se presentan las razones de momios crudas y ajustadas para AT. Los resultados ajustados (por las variables contenidas en el cuadro) muestran que todos los grupos de edad tuvieron más posibilidad de sufrir accidentes de tránsito no fatales que los niños menores de 10 años, aunque los adultos jóvenes (20 a 44 años) fueron los relacionados de modo más notorio ( $R M=3.88$, IC95\%, 2.63-5.71). También se encontró un comportamiento desigual por sexo: los hombres fueron los más afectados al tener $73 \%$ mayor posibilidad de sufrir un AT que las mujeres. Para el área urbana se observó un incremento de la posibilidad de presentar AT ( $R M=1.45 ; I C 95 \%=1.09-1.92)$. Asimismo, los individuos con mayor nivel socioeconómico presentaron mayor posibilidad de accidentarse en el tránsito que los del nivel socioeconómico más bajo, 86\% (NSE alto) y 30\% (NSE medio). Por regiones no se identificaron diferencias estadísticamente significativas. ${ }^{15-23}$

\section{Discusión}

En México, la mayoría de los estudios realizados sobre accidentes de tránsito se ha enfocado en la identificación de los factores de riesgo que incrementan la posibilidad de que un individuo sufra un AT, así como en sus consecuencias en términos de mortalidad y morbilidad y costos de atención médica en grupos de población específica y sólo para algunos estados. ${ }^{5,6,24-32}$ En consecuencia, profundizar en la determinación de la prevalencia de accidentes de tránsito y su distribución geográfica servirá para entender mejor este importante 
Cuadro II

\section{Prevalencias calculadas de accidentes de tránsito no fatales en México por características seleccionadas (ENSANUT 2006, MÉXICO)}

Variable Sin accidente (\%) $\quad$ NoAT $\quad$\begin{tabular}{cc} 
Tipo de accidente de tránsito (AT) \\
\cline { 3 - 4 }
\end{tabular}

Grupos de edad

\begin{tabular}{llrrrrl}
$<10$ años & $19145468(94.83)$ & $979781(4.85)$ & $26510(0.13)$ & $27450(0.14)$ & $10688(0.05)$ & \\
\hline $10-19$ años & $20860458(91.28)$ & $1719924(7.53)$ & $150381(0.66)$ & $47287(0.21)$ & $75657(0.33)$ & \\
\hline $20-44$ años & $34597754(95)$ & $1338535(3.68)$ & $334381(0.92)$ & $75694(0.21)$ & $70857(0.19)$ \\
\hline $45-64$ años & $15503819(94.9)$ & $688575(4.22)$ & $79825(0.49)$ & $46861(0.29)$ & $10078(0.06)$ \\
\hline 65 años o más & $6648108(93.68)$ & $377168(5.31)$ & $12444(0.18)$ & $36162(0.51)$ & $22617(0.32)$ & 0.0000
\end{tabular}

Sexo

$\begin{array}{lrrrrrr}\text { Mujeres } & 51531057(95.37) & 2087762(3.86) & 252839(0.47) & 98789(0.18) & 60323(0.11) & 0.0000\end{array}$

Nivel socioeconómico

\begin{tabular}{|c|c|c|c|c|c|c|}
\hline I más bajo & 40164052 (94.08) & $2188626(5.13)$ & $161760(0.38)$ & $107626(0.25)$ & $67348(0.16)$ & \\
\hline 2 & $24019169(94.15)$ & | $276248(5)$ & $99666(0.39)$ & $59384(0.23)$ & $56557(0.22)$ & \\
\hline 3 & $23569572(94.14)$ & I 165838 (4.66) & 20735 I (0.83) & 48771 (0.19) & $46244(0.18)$ & \\
\hline 4 más alto & $900 I 323(93.31)$ & $47327 \mathrm{I}\left((4.9 \mathrm{I})^{\ddagger}\right.$ & $134764(1.40)^{\S}$ & $17673(0.18)^{\ddagger}$ & $19748(0.20)^{\ddagger}$ & 0.0000 \\
\hline
\end{tabular}

Estrato

\begin{tabular}{|c|c|c|c|c|c|c|}
\hline Rural & 22835029 (94.4I) & I I 97 05। (4.95) & $88652(0.37)$ & $32611(0.13)$ & $33212(0.14)$ & \\
\hline Urbano & 73920578 (93.93) & $3906932(4.96)$ & $514889(0.65)$ & $200843(0.26)$ & I56 $685(0.20)$ & 0.0124 \\
\hline
\end{tabular}

\begin{tabular}{|c|c|c|c|c|c|c|}
\hline \multicolumn{7}{|l|}{ Región } \\
\hline Norte & I8 827823 (94.04) & $981909(4.90)$ & $131792(0.66)$ & $51519(0.26)$ & \multicolumn{2}{|l|}{$27615(0.14)$} \\
\hline Centro & 39296464 (94.05) & $2054889(4.92)$ & $236|6|(0.57)$ & $113706(0.27)$ & \multicolumn{2}{|l|}{$83520(0.20)$} \\
\hline Distrito Federal & 8 I 47544 (94.07) & $406617(4.69)$ & $7764 I(0.90)$ & $14512(0.17)$ & \multicolumn{2}{|l|}{$14968(0.17)$} \\
\hline Sur & 30483776 (94.03) & I 660568 (5.12) & I57 $947(0.49)$ & $53717(0.17)$ & $63794(0.20)$ & 0.2744 \\
\hline Total & 96755607 (94.04) & $5103983(4.96)$ & 603541 (0.59) & $233454(0.23)$ & \multicolumn{2}{|l|}{$189897(0.18)$} \\
\hline \multicolumn{7}{|c|}{$\begin{array}{l}\text { * El estadístico ji cuadrada de Pearson se corrige por el diseño de la encuesta mediante una corrección de segundo ordende Rao y Scott (I984) y conversiór } \\
\text { a un estadístico F. } \\
\text { Prueba de tendencia: }{ }^{\ddagger} p<0.05 ;{ }^{\S} p<0.00 \text { I } \\
\text { \# Valores de } p \text { comparados: sin accidente, no AT y cada tipo de AT }\end{array}$} \\
\hline
\end{tabular}

problema de salud pública y será de utilidad sobre todo para quienes toman decisiones y proponen políticas e intervenciones de salud. La aproximación de un problema específico a través de la epidemiología espacial se ha utilizado en otros problemas de salud y ha demostrado ser de gran valía para la prevención y el diseño de políticas y servicios de salud..$^{27,33-37}$ Sin embargo, el enfoque no debe partir sólo desde el sector salud, ya que la naturaleza multifactorial de los AT requiere que las acciones de promoción y prevención incluyan a otras instituciones incluidas en los AT. En México, por ejemplo, existe una reglamentación que obliga a usar cinturón de seguridad, casco en motociclistas y asientos para infantes, además de que sanciona la utilización de 


\section{Cuadro III}

RegResión LOGísTICA BIVARIADA Y MULTIVARIADA PARA ACCIDENTES DE TRÁNSITO DE ACUERDO CON LAS VARIABLES INDEPENDIENTES EN MÉXICO EN 2006 (ENSANUT 2006)

\begin{tabular}{lll}
\multirow{2}{*}{\begin{tabular}{l} 
Variable \\
Grupos de edad \\
\cline { 2 - 3 }$<10$ años
\end{tabular}} & \multicolumn{2}{c}{ Todos los AT } \\
\cline { 2 - 3 } $\begin{array}{ll}\text { I0-19 años } \\
\text { 20-44 años }\end{array}$ & $3.88(2.63-5.73)^{*}$ & $3.82(2.59-5.62)^{*}$ \\
\hline $45-64$ años & $4.12(2.76-6.13)^{*}$ & $3.88(2.63-5.71)^{*}$ \\
\hline 65 y más años & $2.61(1.67-4.08)^{*}$ & $2.36(1.51-3.71)^{*}$ \\
\hline
\end{tabular}

Sexo

\begin{tabular}{lll} 
Mujeres & $\mathrm{I}$ & $\mathrm{I}$ \\
\hline Hombres & $\mathrm{I} .70(\mathrm{I} .3 \mathrm{I}-2.2 \mathrm{I})^{*}$ & $\mathrm{I} .73(\mathrm{I} .33-2.25)^{*}$
\end{tabular}

Nivel socioeconómico

\begin{tabular}{lll} 
I más bajo & I & I \\
\hline 2 & $1.07(0.81-1.42) \mathrm{n} / \mathrm{s}$ & $0.99(0.74-1.32) \mathrm{n} / \mathrm{s}$ \\
\hline 3 & $1.53(1.19-1.96)^{\ddagger}$ & $1.30(1.00-1.70)^{\S}$ \\
\hline 4 más alto & $2.28(1.51-3.45)^{*}$ & $1.86(1.19-2.91)^{\ddagger}$
\end{tabular}

Estrato

\begin{tabular}{lll} 
Rural & $\mathrm{I}$ & $\mathrm{I}$ \\
\hline Urbano & $\mathrm{I} .74(1.34-2.28)^{*}$ & $\mathrm{I} .45(1.09-1.92)^{\#}$
\end{tabular}

Región

\begin{tabular}{lll} 
Norte & $\mathrm{I} .24(0.98-\mathrm{I} .57)^{\S}$ & $0.98(0.76-1.26) \mathrm{n} / \mathrm{s}$ \\
\hline Centro & $\mathrm{I} .22(0.93-1.60) \mathrm{n} / \mathrm{s}$ & $\mathrm{I} .07(0.83-1.39) \mathrm{n} / \mathrm{s}$ \\
\hline Distrito Federal & $\mathrm{I} .45(0.95-2.23)^{\S}$ & $\mathrm{I} .07(0.70-1.64) \mathrm{n} / \mathrm{s}$ \\
\hline Sur & $\mathrm{I}$ & $\mathrm{I}$
\end{tabular}

$* p<0.001$

$\ddagger p<0.01$

$\S p<0.10$

$\# p<0.05$

Nota: la variable dependiente se categoriza como: $0=$ sujetos sin ningún tipo de accidente y I= sujetos que notificaron tener AT. En el modelo multivariado las razones de momios están ajustadas por las demás variables contenidas en el cuadro

N/S: No significativo

medios de comunicación mientras se conduce y manejar bajo el efecto del alcohol y otras sustancias tóxicas. ${ }^{38}$

Los hallazgos del presente estudio permiten confirmar la ya conocida importancia de los accidentes en general y de manera particular de los AT en México. La alta prevalencia de estos últimos es un llamado de atención a los responsables de formular políticas vin- culadas con los AT. Un porcentaje de $16.7 \%$ señala que una de cada seis personas accidentadas es víctima de un AT. Sin embargo, a pesar de que en fecha reciente la Commission for Global Road Safety resaltó que la mayoría de las muertes por AT (85\%) ocurre en países de bajos ingresos, ${ }^{39,40}$ y aun cuando representa una buena parte de la carga de morbimortalidad, este tema no se ha incluido en las metas de desarrollo del milenio por los países más ricos. ${ }^{40}$ Es evidente que el proceso de urbanización que acompaña al desarrollo económico en las ciudades crea condiciones que tienden a incrementar el riesgo de accidentes, ${ }^{4}$ por lo que es necesaria la institución de acciones dirigidas a contrarrestar tales riesgos. Por otro lado, se ha destacado que las muertes por AT en el mundo tienen una distribución desigual entre las zonas rural y urbana, ${ }^{4,27}$ en la que se observa que $77 \%$ de las muertes ocurre en el área urbana, lo cual es consistente con los hallazgos de este estudio en el que también son las áreas urbanas las que muestran las prevalencias más altas: hasta $85 \%$ de AT tiene lugar en áreas urbanas. Sin embargo, es necesario resaltar que este estudio alude tan sólo a AT no fatales. El fenómeno de urbanización y AT se ha documentado con anterioridad y se ha relacionado con el mejoramiento de la potencia de los coches y, por lo tanto, con el aumento del riesgo potencial de accidente y muerte por las altas velocidades. ${ }^{39}$ Otros factores que se han señalado son el incremento de la concentración de vehículos y personas que coexisten en las grandes ciudades y el rápido crecimiento, industrialización y falta de planeación de la urbanización.

En cuanto a la distribución geográfica de los AT, se observó que los estados con mayor desarrollo presentan mayor prevalencia de AT. Así, Jalisco, Aguascalientes y Sonora registran los mayores porcentajes de AT, en tanto que en dos de los estados más pobres del país (Guerrero y Oaxaca, junto con Michoacán) existen las menores prevalencias. Esto podría atribuirse a que los primeros estados tienen: a) mayor cantidad de parque vehicular; b) mayor número de carreteras y autopistas, lo cual incrementa la velocidad de desplazamiento de los automóviles; c) mejoras en los motores de los automóviles, que los hace más veloces; d) mayor concentración de personas por actividades laborales en las ciudades, que supone una exposición más frecuente a los AT. Este hallazgo es congruente con un estudio de Van Beek y colaboradores ${ }^{41}$ en 21 países miembros de la OCDE; dichos autores demostraron en el plano ecológico que la mortalidad por accidentes de tránsito y el nivel de prosperidad (dólares per cápita) se vinculan de manera positiva; cuando aumenta el nivel de prosperidad lo hacen también los accidentes. No obstante, también advirtieron que en algunos países se puede llegar un nivel de prosperidad en el que los accidentes 
comienzan a disminuir. En este sentido, un estudio ${ }^{42}$ reciente demuestra un comportamiento desigual de los AT en los países desarrollados y en desarrollo; en los primeros se observa una relación negativa, mientras que en los segundos la relación es positiva. Sin embargo, los resultados son contradictorios con los resultados de un estudio realizado en Pensilvania, en el que los autores observaron en términos ecológicos que los poblados con mayor porcentaje de pobres presentaron mayor riesgo de $\mathrm{AT}_{,}^{43}$ lo cual es consistente con la aseveración del párrafo anterior. En el análisis de AT y NSE individual se observó que las personas con NSE mejor sufrían más choques y aquéllas con el peor NSE eran propensas a ser atropelladas. Esto coincide con estudios previos; por ejemplo, Hasselberg y colaboradores ${ }^{44}$ observaron que el riesgo para peatones aumentó $20 \%$ en quienes tuvieron peores salarios. En otro estudio, ${ }^{45}$ los mismos autores señalaron que los conductores de NSE bajo presentan lesiones más graves que los de NSE alto. Stevenson y colaboradores ${ }^{46}$ también publicaron una relación inversa entre el NSE y el riesgo de lesión en peatones. Estos resultados se relacionan en particular con el acceso a los automóviles. La gente con más recursos puede optar por la compra de automóvil; en cambio, los más pobres se trasladan a pie con frecuencia para abordar los servicios públicos de transporte y ello los hace más vulnerables a un atropellamiento.

La pérdida de la vida es la consecuencia más importante de cualquier problema de salud, más aún cuando se vincula con un suceso catastrófico como los accidentes de tránsito. Éstos, en virtud de su propia naturaleza, traen consigo pérdidas importantes para las familias y la sociedad. Por esa razón, muchos de los estudios orientados a conocer la magnitud de los AT se basan en los registros oficiales de la mortalidad, lo que permite identificar la carga de los AT sólo en esos términos. Otros estudios analizan la magnitud de los AT a partir de los registros hospitalarios, de tal forma que es posible conocer la magnitud de lesionados que exige atención hospitalaria, pero debido a que todavía no se generaliza la práctica de notificar la causa externa de las lesiones, además de que no todos los lesionados por AT demandan atención médica, existe una notable subestimación del número real de accidentados. Por estas razones, los estudios futuros deberán incluir tanto los datos de encuestas poblacionales como el análisis basado en datos hospitalarios y de mortalidad. Ello permitirá tener un panorama epidemiológico más completo de los AT en México. ${ }^{15}$ Éste es el primer estudio que publica cálculos nacionales sobre los distintos tipos de accidentes de tránsito no-fatales por grupo de edad, sexo, nivel socioeconómico, lugar de residencia y distribución nacional.
Las características sociodemográficas (edad y sexo) de las personas que sufrieron AT son consistentes con otros trabajos realizados sobre mortalidad y morbilidad por AT: ${ }^{16-21}$ los individuos que mueren, se discapacitan o se lesionan más son los hombres en edad productiva. Esta caracterización también coincide con el Reporte Mundial de Salud ${ }^{4}$ y las notificaciones de diferentes países. ${ }^{16}$ Este punto se explica porque estos grupos tienen que desplazarse a sus centros de trabajos y por tanto están más expuestos. Este fenómeno se observa a pesar de la fuerte presencia de las mujeres en el proceso laboral, que supone también desplazarse dentro de las ciudades. Otros factores pueden intervenir y se relacionan en particular con la condición de género, esto es, quién maneja y de qué forma lo hace.

Los resultados también coinciden con los informes de Markogiannakis y colaboradores y Kong y colaboradores, ${ }^{22,23}$ según los cuales los atropellamientos son más comunes en los grupos de población de mayor edad, además de que también en ellos se identifican las lesiones más graves.

A pesar de la importancia de los resultados, es necesario considerar algunos aspectos que limitan la comparación. Algunas variables de interés no se incluyeron en el presente análisis por falta de información, entre ellas consumo de alcohol y medicamentos. Este estudio considera el autorreporte, que si bien es la forma en la que se realizan las encuestas nacionales, la falta de validación puede introducir sesgos de reporte en el estudio, ya que es de esperar que aquellos AT percibidos como no graves se olviden con mayor frecuencia. Si bien la información generada mediante autorreporte puede tener limitaciones, su uso ha demostrado ser una medida útil, válida y confiable para conocer diferentes aspectos relacionados con salud, ya que es posible recabar información de grandes grupos poblacionales con mayor capacidad de inferencia que los estudios clínicos; asimismo, contar de manera simultánea con gran cantidad de información socioeconómica y demográfica permite identificar ciertos patrones sistemáticos de enfermedad entre diferentes grupos poblacionales, lo cual facilita la identificación de factores de riesgo. ${ }^{47-51}$

Los resultados de este estudio, pese a las limitaciones señaladas, constituyen una excelente aproximación a una visión diferente de este importante problema de salud en México. Dado que los AT representan poco más de un millón de lesionados, de los cuales cerca de $80 \%$ exigió algún tipo de atención por año, resulta cada vez más urgente aplicar medidas eficaces encaminadas a su reducción. Estas intervenciones deben considerar la distribución espacial, las características y necesidades específicas y el nivel de desarrollo de cada estado de la República. Además, el carácter multifactorial de los AT 
requiere que las acciones de promoción y prevención se diseñen e instituyan de forma multisectorial, multidisciplinaria e interinstitucional.

\section{Referencias}

I.World Health Organization. International Statistical Classification of Diseases and Related Health Problems 10th Revision Version for 2007. [consultado 2007 octubre 17]. Disponible en: http://www.who.int/ classifications/apps/icd/icdIOonline/.

2. Krug EG, Sharma GK, Lozano R. The global burden of injuries. Am J Public Health 2000;90:523-526.

3. Organización Mundial de la Salud. Día mundial de la salud 2004. Objetivos y mensajes del día mundial de la salud 2004. OMS; 2004. [consultado 2005 abril]. Disponible en: Url: Http://www.who.int/worldhealth-day/2004/toolkit/objectives/es/.

4. World Health Organization. World report on road traffic injury prevention. Geneva:WHO, 2004.

5. Hijar M, Chu Ld, Graus J. Cross national comparison of injury mortality: Los Angeles County, California and Mexico City, Mexico. Int J Epidemiol 2000;29:7|5-72I.

6. Hijar M, Graus J,Tovar V, Carrillo C.Analisis of fatal pedestrian injuries in Mexico City, 1994-1997. Injury 2001;32(4):279-284.

7. Hijar M. Prevención de accidentes de tránsito en México: qué funciona y qué no. En: Hijar M,Vázquez E, comp. Foro nacional sobre accidentes de tránsito en México. Enfrentando los retos a través de una visión intersectorial. México 2003:23-31.

8. Lozano A. Lesiones por vehículos en México. Fuentes y sistemas de información existentes. En: Hijar M,Vázquez E, comp. Foro nacional sobre accidentes de tránsito en México. Enfrentando los retos a través de una visión intersectorial. México 2003:15-21.

9.Vázquez-Vela E. Peso de las lesiones debidas a accidentes de tránsito. En: Hijar M,Vázquez E, comp. Foro nacional sobre accidentes de tránsito en México. Enfrentando los retos a través de una visión intersectorial. México 2003:II-14.

10. Secretaría de Prevención y Protección de Salud. México: Secretaría de Salud. Programa de Acción:Accidentes, 2002.

I I. Olaiz-Fernández G, Rivera-Dommarco J, Shamah-Levy T, Rojas R, Villalpando-Hernández S, Hernández-Avila M, Sepúlveda-Amor J. Encuesta Nacional de Salud y Nutrición 2006. Cuernavaca, México: Instituto Nacional de Salud Pública, 2006.

12. Consejo Nacional de Población. Metodología de estimación del índice de marginación. Anexo C. México: CONAPO, 2005. [consultado 2006 octubre]. Disponible en: http://www.conapo.gob.mx/publicaciones/ margina2005/anexo_c.pdf.

13. Rao JNK, Scott AJ. On chi-squared tests for multi-way contingency tables with cell proportions estimated from survey data.Ann Statist 1984;12:46-60

14. Sun GW, Shook TL, Kay GL. Inappropriate use of bivariable analysis to screen risk factors for use in multivariable analysis. J Clin Epidemiol 1996;49:907-916

I5. Trinh Van H, Singhasivanon P, Kaewkungwal J, Suriyawongpaisal P, Hoang Khai L. Estimation of non fatal road traffic injuries in Thai Nguyen,Vietnam using capture-recapture method. South Asian J Trop Med Public Health 2006;37:405-4II.

16. Ganveer BG, Tiwari RR. Injury paterrn among non-fatal road traffic accident cases: a cross-sectional study in central India. Indian J Med Sci 2005;59:9-12.
17. Saiz-Sánchez C, Bautista-Rentero D, Corella-Piquer D, Cortina-Birlanga S, González-Arraez Jl.Análisis edad-periodo-cohorte de la mortalidad por accidentes de tráfico en España. Salud Publica Mex 1999;41:170-176.

18. Guly CM, Guly HR, Bouamra O, Gray RH, Lecky FE. Ocular injuries in patients with major trauma. Emerg Med J 2006;23:915-917.

19. Soffer D, Zmora O, Klausner JB, Szold O, Givon A, Halpern P, et al. Alcohol use among trauma victims admitted to a level I trauma center in Israel. Isr Med Assoc J 2006;8:98-102.

20.Vorko-Jovic A, Kern J, Biloglav Z. Risk factors in urban road traffic accidents. J Safety Res 2006;37:93-98.

21. Meneses-González F, Rea R, Ruiz-Matus C, Hernández-Ávila M. Accidentes y lesiones en cuatro hospitales del Distrito Federal. Salud Publica Mex 1993;35:448-455.

22. Markogiannakis H, Sanidas E, Messaris E, Koutentakis D,Alpantaki K, Kafetzakis A, et al. Motor vehicle trauma: analysis of injury profiles by road-user category. Emerg Med J 2006;23:27-3I.

23. Kong LB, Lekawa M, Navarro RA, McGrath J, Cohen M, Margulies DR, et al. Pedestrian-motor vehicle trauma: an analysis of injury profiles by age. J Am Coll Surg 1996;182:17-23.

24. Hidalgo-Solorzano EC, Hijar M, Blanco-Muñoz J, Kageyama-Escobar ML. Severity of injuries in public streets of an urban area in Mexico. Salud Publica Mex 2005;47:30-38.

25. Hijar-Medina MC,Arredondo A, Carrillo C, Solorzano L. Road traffic injuries in an urban area in Mexico.An epidemiological and costs analysis. Accid Anal Prev 2004;36:37-42.

26. Hijar-Medina MC, Flores-Aldana ME, López-López MV. Cinturón de seguridad y gravedad de lesiones en accidentes de tráfico en carretera. Salud Publica Mex 1996;38: I 8-127.

27. Hijar-Medina MC. Utilidad del análisis geográfico en el estudio de las muertes por atropellamiento. Salud Publica Mex 2000;42:188-193. 28. Hijar-Medina MC, Tapia-Yánez JR, López-López MV, Solórzano-Flores LI, Lozano-Ascencio R. Factores de riesgo de accidentes en niños. Estudio de casos y controles. Bol Med Hosp Infant Mex 1993;50:463-474.

29. Hijar-Medina M, Carrillo-Ordaz C, Flores-Aldana M,Anaya R, López-López M. Factores de riesgo de lesión por accidentes de tráfico y el impacto de una intervención sobre la carretera. Rev Saude Publica 1999;33:505-512.

30. Hijar-Medina MC, Trostle J, Bronfman M. Pedestrian injuries in Mexico: a multi-method approach. Soc Sci Med 2003;57:2149-2159.

31. Hijar M, Kraus J,Tovar V, Carrillo C.Analysis of fatal pedestrian injuries in Mexico City, 1994-1997. Injury 200 I;32:279-284.

32. Celis A, Gómez-Lomelí Z,Armas J.Tendencias de mortalidad por traumatismos y envenenamientos en adolescentes. México, 1979-1997. Salud Publica Mex 2003;45:8-15.

33. Minaker LM, McCargar L, Lambraki I, Jessup L, Driezen P, Calengor $\mathrm{K}$, et al. School region socio-economic status and geographic locale is associated with food behaviour of Ontario and Alberta adolescents. Can J Public Health 2006;97:357-36I.

34. Lapa TM,Albuquerque MF, Carvalho MS, Silveira Junior JC. Spatial analysis of leprosy cases treated at public health care facilities in Brazil. Cad Saude Publica 2006;22:2575-2583.

35. Siffel C, Strickland MJ, Gardner BR, Kirby RS, Correa A. Role of geographic information systems in birth defects surveillance and research. Birth Defects Res A Clin Mol Teratol 2006;76:825-833.

36. Olson KL, Grannis SJ, MandI KD. Privacy protection versus cluster detection in spatial epidemiology. Am J Public Health 2006;96:2002-2008. 37. Kazembe LN, Kleinschmidt I, Holtz TH, Sharp BL. Spatial analysis and mapping of malaria risk in Malawi using point-referenced prevalence of infection data. Int J Health Geogr 2006;5:4I.

38. Consejo de Salubridad General.Acuerdo por el que se establecen diversas medidas de protección tendientes a disminuir la ocurrencia y el impacto de los accidentes de tránsito. Diario Oficial. México DF: Noviembre 19, 2003. 
39. Perel P, McGuire M, Eapen K, Ferraro A. Research on preventing road traffic injuries in developing countries is needed. BMJ 2004;328:895. 40. Editorial.The hidden epidemic of road-traffic injuries. Lancet 2006;367:1954.

4I. Beeck EF, Borsboom GJ, Mackenbach JP. Economic development and traffic accident mortality in the industrialized world, 1962-1990. Int J Epidemiol 2000;29:503-9.

42. Bishai D, Quresh A, James P, Ghaffar A. National road casualties and economic development. Health Econ 2006;15:65-8I.

43. Aguero-Valverde J, Jovanis PP. Spatial analysis of fatal and injury crashes in Pennsylvania. Accid Anal Prev 2006;38:618-625.

44. Hasselberg M, Laflamme L,Weitoft GR. Socioeconomic differences in road traffic injuries during childhood and youth: a closer look at different kinds of road user.J Epidemiol Community Health 2001;55:858-862. 45. Hasselberg M, Vaez M, Laflamme L. Socioeconomic aspects of the circumstances and consequences of car crashes among young adults. Soc Sci Med 2005;60:287-295.

46. Stevenson MR, Jamrozik KD, Spittle J.A case-control study of traffic risk factors and child pedestrian injury. Int J Epidemiol 1995;24:957-964.
47. Dunn JR, Walker JD, Graham J,Weiss CB. Gender differences in the relationship between housing socioeconomic status, and self-reported health status. Rev Environ Health 2004; 19:177-195.

48. Borrell C, Muntaner C, Benach J,Artazcoz L. Social class and selfreported health status among men and women: what is the role of work organisation household material standards and household labour? Soc Sci Med 2004;58:1869-1887.

49. McNeely MJ, Boyko EJ. Diabetes-related comorbidities in Asian Americans Results of a National Health Survey.J Diabetes Complicat 2005; 19:101-106.

50. Ávila-Burgos L, Ramírez-Valverde G, Martínez-Damián MACruz-Valdez A, Santiago-Cruz MJ, Medina-Solís CE. Socio-economic determinants of inequality and self-reported morbidity among adolescents in a developing country. Saudi Med J 2005;26:447-453.

5I. Bjorvatn B, Sagen IM, Oyane N,Waage S, Fetveit A, Pallesen S, et al. The association between sleep duration body mass index and metabolic measures in the Hordaland Health Study.J Sleep Res 2007;16:66-76. 